\title{
Microaggressions in Medicine
}

\author{
Brittany Feaster, MHS; Lynn McKinley-Grant, MD, MA; Amy J. McMichael, MD
}

\section{PRACTICE POINTS}

- As providers, we must strive to understand all categories of racism and expose the many ways prejudice manifests within medical training and clinical practice.

- Intervention strategies must be implemented to reduce the likelihood of the occurrence of microaggressions in medicine and challenge the stereotypes that undergird implicit bias.

- It is important to promote collaboration in diversity, equity, and inclusion efforts to demonstrate support for women and underrepresented minority medical students, residents, physicians, providers, and patients.

Microaggressions are behaviors that stem from implicit bias and occur at an interpersonal level. In medicine, microaggressions may be encountered both in training and clinical practice. Although often unintentional or unconscious by the offender, microaggressions are harmful to the health and safety of women and underrepresented minority (URM) medical students, residents, physicians, other providers, and patients. This article aims to define microaggressions, present example scenarios, and provide discourse regarding microaggressions within the framework of medicine.

Cutis. 2021;107:235-237.

A s manifestations of overt racism and macroaggressions have gained increased visibility, there is a need for discussion of another expression of racism: microaggressions. Although racism classically is viewed as blatant structural, attitudinal, and behavioral prejudice, experts pose that the face of racism has evolved into a more covert insidious form. This form of racism was originally coined racial microaggressions by psychiatrist Chester M. Pierce, MD, 50 years ago., ${ }^{1,2}$ Since that time, microaggressions have further expanded to describe "brief and commonplace daily verbal, behavioral, and environmental indignities, whether intentional or unintentional, that communicate hostile, derogatory, or negative racial, gender, sexual-orientation, and religious slights and insults to the target person or group." ${ }^{3}$ This article aims to define and depict examples of microaggressions in medicine, discuss the resulting harmful effects, and offer strategies to minimize and counter these negative ramifications.

\section{What are microaggressions?}

Microaggressions are behaviors that stem from implicit bias and occur at an interpersonal level. Implicit bias refers to unconscious stereotypes, assumptions, and beliefs held about an individual's identity. One of the earliest microaggressions-invisibility-was characterized by Ralph Ellison in his novel Invisible Man. Ellison states, "I am invisible, understand, simply because people refuse to see me ... When they approach me they see only my surroundings, themselves, or figments of their imagination-indeed, everything and anything except me." ${ }^{\prime 4}$ This concept of invisibility is a primary microaggression faced by people of color.

In medicine, microaggressions and implicit bias may be encountered throughout medical training and clinical practice in interactions with colleagues, superiors, patients, and patients' families., ${ }^{5,6}$ Examples of microaggressions in medicine include demeaning comments, nonverbal disrespect, generalizations of social identity, assumption of nonphysician status, role- or credential-questioning behavior, explicit epithets, rejection of care, questioning or inquiries of ethnic/racial origin, and sexual harassment. ${ }^{7}$

An example of microaggressions in medicine was fully displayed when physician Tamika Cross described her experience of being turned away from helping an unresponsive passenger during a flight emergency.

[T] he flight attendant yells"call overhead for a physician on board." I raised my hand to grab her attention. She said to me"oh no sweetie put [your] hand down, we are looking for actual physicians or nurses or some type of medical personnel, we don't have time to talk to you" . . . Another "seasoned" white male approaches the row and says he is a physician as well. She says to me"thanks for your help but he can help us, and he has his credentials." ${ }^{8}$

Ms. Feaster and Dr. McMichael are from the Department of Dermatology, Wake Forest School of Medicine, Winston-Salem, North Carolina. Dr. McKinley-Grant is from the Department of Dermatology, Howard University College of Medicine Hospital, Washington, DC.

The authors report no conflict of interest.

Correspondence: Amy J. McMichael, MD, 4618 Country Club Rd, Winston-Salem, NC 27104 (amcmicha@wakehealth.edu). doi:10.12788/cutis.0249 


\section{What are the effects of microaggressions?}

Although microaggressions may be unconscious and unintentional by the offender, the negative ramifications are notable. Recent studies report that women and underrepresented minority (URM) medical students, residents, and physicians experience microaggressions and implicit bias at a higher prevalence and frequency compared with their male and non-URM counterparts. ${ }^{7,9}$ Repetitive microaggressions are harmful to the health and safety of women and URM medical students, residents, physicians, other providers, and patients. The Table provides example scenarios of microaggressions in medicine categorized according to Berk. ${ }^{10}$

Microaggressions negatively impact physical, mental, and emotional well-being. Current data support that medical students and residents who experience microaggressions are more likely to report associated symptoms of burnout, depression, and suicidal thoughts. ${ }^{11,12}$ Subjection to persistent bias can lead to minority status stress and racial battle fatigue, creating feelings of invisibility, isolation, exclusion, and loneliness for those impacted. ${ }^{13,14}$

In the book Black Man in a White Coat: A Doctor's Reflections on Race and Medicine, Damon Tweedy, MD, reflects on race in medicine. Tweedy notes his experience as a medical student when a professor mistakenly assumed he was a maintenance worker in the classroom. Tweedy describes how he internalized the exchange and, despite his success throughout the course of his medical training, combatted feelings of anxiety, self-doubt, and implied inferiority. ${ }^{15}$

Although microaggressions are harmful to one's health, they also undermine the learning and teaching experience for students, residents, and faculty, and they detract from the larger goal of providing care for patients. ${ }^{11}$ Frequent devaluing and questioning of an individual's contributions, qualifications, and credentials

\section{Consequences of Microaggressions in Medicine}

\section{Example scenarios in medicine \\ Exclusion from a monthly meeting of colleagues with superior or department chair \\ No administrative assistance while other colleagues have assistance \\ Repeatedly not having a resident or medical student assigned to you \\ Being asked to provide more letters of recommendation than other colleagues; assuming a female or URM physician is a nurse or maintenance worker \\ Being labeled as disrespectful by staff or superiors for not using "ma'am" or "sir" when speaking to patients \\ Being told that only 1 person can apply for a grant and that another faculty member has already been selected \\ Being singled out for comments or asked to lead by colleagues or superiors whenever issues concerning race or diversity arise; given excess responsibilities on department and university committees for diversity}

Having a suggestion not recognized when presented at a meeting with colleagues but acknowledged when presented a few minutes later by male or non-URM colleague

Faculty, residents, medical students, and physicians experience burnout, depression, and PTSD due to repeatedly experiencing and witnessing microaggressions in the academic setting and workplace

Staring or not turning around to speak when a woman or URM provider walks into the office; overhearing a receptionist say to a patient, "I want to warn you, she is Black"
Berk's law: consequences of microaggressions ${ }^{10}$

Creating feelings of isolation, exclusion, loneliness, and tokenism

Lowering the individual's work productivity and problem-solving abilities

Devaluing the individual's research, scholarship, and teaching contributions

Undermining and questioning the individual's qualifications and credentials Subjecting the individual to biased and unfair reviews for performance appraisal, tenure, merit pay, and awards

Excluding the individual from grants, research projects, team teaching, mentoring, guest lectures, and professional conferences

Committing the individual to excess service on diversity, task force, department, and university committees as the face of diversity

Resulting in feelings of being ignored, overlooked, unappreciated, underrespected, undercompensated, overworked, misrepresented, and devalued

Producing physical and mental health problems, such as depression, frustration, anger, rage, low self-esteem, stress, PTSD, anxiety, substantial weight gain, high blood pressure, and cardiovascular disease

Creating an unwelcome, hostile, and invalidating climate that is alienating, stressful, polarized, and risky

Abbreviations: URM, underrepresented minority; PTSD, posttraumatic stress disorder. 
based on identity can lower productivity and problemsolving abilities. These behaviors cultivate an unwelcome and hostile work/learning environment that is stressful and polarizing for the recipient.

Despite the heavy burden of microaggressions, most students, residents, and faculty physicians do not report incidents to their institutions and feel that training, resources, and policies to respond to bias adequately are lacking. ${ }^{7}$ As a result of implicit bias and microaggressions, women and URM medical students and providers are unable to focus solely on the practice of medicine. They are tasked with the additional burden of shouldering the emotional and cognitive complexities that microaggressions produce. ${ }^{16}$

\section{What are strategies to reduce microaggressions in medicine?}

To minimize the harmful effects of microaggressions, intervention strategies must be implemented that reduce the likelihood of the occurrence of microaggressions and challenge the stereotypes that undergird implicit bias. These strategies include cultivating allies, followed by demanding structural accountability. Allies are members of the majority group who collectively collaborate with members of the nonmajority group to effect change through the promotion of diversity, equity, and inclusion efforts. ${ }^{17}$ Cultivating allies involves building a network of collaboration among these groups and emphasizes education. Education is critical for allies to address microaggressions at the interpersonal level. This process of education involves personal reflection and self-awareness in exploring one's biases, fears, and assumptions. Integral to this step is broadening one's acceptance of different cultures, racial/ethnic groups, and identities. There must be a willingness to engage in difficult or uncomfortable conversations and a readiness to actively listen to concerns rather than perpetuating further harm through avoidance and dismissive or defensive behavior. ${ }^{18}$

Demanding structural accountability facilitates deconstruction of bias and microaggression at the larger systemic level. This strategy involves implicit bias and antiracism training, development of retention plans, and identification of mentors for women and URM providers and students. Implicit bias and microaggression training and policies should be incorporated into medical education and resident curriculums. Similarly, educational resources and training must be made available to practicing physicians, faculty, and other providers through their institutions and places of employment. Equipping students and providers with the tools needed when microaggressions are witnessed or experienced demonstrates systemic-level accountability and communicates the importance of the issue. Furthermore, the development of retention plans and identification of mentors provide a support system and foster a culture of inclusion where recipients of microaggressions feel protected and valued. Increased feelings of inclusivity and belonging help bridge the gap created through microaggressions and implicit bias.

\section{Final Thoughts}

Despite an often covert nature, the detrimental effects of microaggressions are tangible and far reaching. As providers, we must strive to understand all categories of racism and expose the many ways prejudice manifests within medical training and clinical practice. It is our obligation to undertake the challenge of "making the 'invisible' visible" as we confront microaggressions and implicit bias to promote a safer and more inclusive medical community and workforce. ${ }^{19}$

\section{REFERENCES}

1. Torres MB, Salles A, Cochran A. Recognizing and reacting to microaggressions in medicine and surgery. JAMA Surg. 2019;154:868-872. doi:10.1001/jamasurg.2019.1648

2. Williams MT. Microaggressions: clarification, evidence, and impact. Perspect Psychol Sci. 2020;15:3-26. doi:10.1177/1745691619827499

3. Sue DW. Microaggressions in Everyday Life: Race, Gender, and Sexual Orientation. Wiley; 2010.

4. Ellison R. Invisible Man. Random House; 1952.

5. Molina MF, Landry AI, Chary AN, et al. Addressing the elephant in the room: microaggressions in medicine. Ann Emerg Med. 2020;76:387-391. doi:10.1016/j.annemergmed.2020.04.009

6. Overland MK, Zumsteg JM, Lindo EG, et al. Microaggressions in clinical training and practice. PM R. 2019;11:1004-1012. doi:10.1002/pmrj.12229

7. de Bourmont SS, Burra A, Nouri SS, et al. Resident physician experiences with and responses to biased patients. JAMA Netw Open. 2020;3:e2021769. doi:10.1001/jamanetworkopen.2020.21769

8. TK Cross Facebook page. October 9, 2016. Accessed April 19, 2021. https://www.facebook.com/tamika.cross.52/posts/658443077654049

9. Periyakoil VS, Chaudron L, Hill EV, et al. Common types of genderbased microaggressions in medicine. Acad Med. 2020;95:450-457. doi:10.1097/ACM.0000000000003057

10. Berk RA. Microaggressions trilogy: part 1 . why do microaggressions matter? J Fac Dev. 2017;31:63-73.

11. Chisholm LP, Jackson KR, Davidson HA, et al. Evaluation of racial microaggressions experienced during medical school training and the effect on medical student education and burnout: a validation study. I Natl Med Assoc. 2020:S0027-9684(20)30428-4. doi:10.1016/j.jnma.2020.11.009

12. Hu YY, Ellis RJ, Hewitt DB, et al. Discrimination, abuse, harassment, and burnout in surgical residency training. N Engl J Med. 2019; 381:1741-1752. doi:10.1056/NEJMsa1903759

13. Acholonu RG, Oyeku SO. Addressing microaggressions in the health care workforce-a path toward achieving equity and inclusion.JAMANetw Open. 2020;3:E2021770. doi:10.1001/jamanetworkopen.2020.21770

14. O'Keefe VM, Wingate LR, Cole AB, et al. Seemingly harmless racial communications are not so harmless: racial microaggressions lead to suicidal ideation by way of depression symptoms. Suicide Life Threat Behav. 2015;45:567-576. doi:10.1111/sltb.12150

15. Tweedy D. Black Man in a White Coat: A Doctor's Reflections on Race and Medicine. Picador; 2016.

16. Osseo-Asare A, Balasuriya L, Huot SJ, et al. Minority resident physicians' views on the role of race/ethnicity in their training experiences in the workplace. JAMA Netw Open. 2018;1:E182723. doi: 10.1001/jamanetworkopen.2018.2723

17. Melaku TM, Beeman A, Smith DG, et al. Be a better ally. Harvard Business Review. Published November-December 2020. Accessed April 23, 2021. https://hbr.org/2020/11/be-a-better-ally

18. Sue DW, Capodilupo CM, Torino GC, et al. Racial microaggressions in everyday life: implications for clinical practice. Am Psychol. 2007;62:271-286. doi:10.1037/0003-066X.62.4.271

19. Sue DW. Whiteness and ethnocentric monoculturalism: making the "invisible" visible. Am Psychol. 2004;59:761-769. doi:10.1037/0003-066X.59.8.761 